\title{
A Proof of Lojasiewicz's Theorem
}

\author{
Namhoon Kim \\ Department of Mathematics Education, Hongik University, 72-1 Sangsu-dong, Mapo-gu, Seoul 121-791, Republic of Korea \\ Correspondence should be addressed to Namhoon Kim; nkim@hongik.ac.kr
}

Received 28 April 2014; Accepted 18 August 2014; Published 11 September 2014

Academic Editor: Gerassimos Barbatis

Copyright (C) 2014 Namhoon Kim. This is an open access article distributed under the Creative Commons Attribution License, which permits unrestricted use, distribution, and reproduction in any medium, provided the original work is properly cited.

We give a necessary and sufficient condition for a primitive of a distribution to have the value at a point in the sense of Łojasiewicz. A formula defining the indefinite integral of a distribution with a basepoint is introduced, and further structural results are discussed.

\section{Introduction}

Let $\mathscr{D}=\mathscr{D}(\mathbb{R})$ be the topological $\mathbb{C}$-vector space of complex valued compactly supported test functions on $\mathbb{R}$, and let $\mathscr{D}^{\prime}=$ $\mathscr{D}^{\prime}(\mathbb{R})$ be the space of complex valued distributions on $\mathbb{R}$. In the following discussion, a distribution $f \in \mathscr{D}^{\prime}$ is also denoted by $f(x)$, and the dual pairing between $f \in \mathscr{D}^{\prime}$ and a test function $\phi \in \mathscr{D}$ is denoted by either $\langle f, \phi\rangle$ or $\langle f(x), \phi(x)\rangle$. On the other hand, the letter $x_{0}$ will always denote a point.

According to Łojasiewicz [1], a distribution $f \in \mathscr{D}^{\prime}$ has the value $c \in \mathbb{C}$ at $x_{0}$ if

$$
f\left(a x+x_{0}\right) \longrightarrow c
$$

in $\mathscr{D}^{\prime}$ as $a \rightarrow 0$. If such a value $c$ exists at $x_{0}$, we will say that $f$ is evaluable at $x_{0}$ and write $f\left(x_{0}\right)=c$. For $f$ to be evaluable at $x_{0}$, it suffices for $\lim _{a \rightarrow 0} f\left(a x+x_{0}\right)$ to exist in $\mathscr{D}^{\prime}$, as the limit can only be a constant. We can equivalently require that there exists $c \in \mathbb{C}$ such that $\lim _{a \rightarrow 0^{+}} f\left(a x+x_{0}\right)=c$, as this entails $\lim _{a \rightarrow 0^{-}} f\left(a x+x_{0}\right)=c$. Simply requiring the existence of $\lim _{a \rightarrow 0^{+}} f\left(a x+x_{0}\right)$ does not suffice, as the limit may in general be of the form $c_{1}+c_{2} H\left(x-x_{0}\right)$, where $H$ is the Heaviside step function.

One interesting consequence of this definition is the following.

Theorem 1 (Łojasiewicz). If a distribution $f$ is evaluable at $x_{0}$, then any primitive $F$ of $f$ is also evaluable at $x_{0}$.

This result is useful in various circumstances. For instance, if a distribution $f$ is evaluable at $a$ and $b$, then so is any primitive $F$ of $f$, and we may define a definite integral of $f$ as

$$
\int_{a}^{b} f=F(b)-F(a) .
$$

These ideas are connected with an interesting construction of distributional integral in the work of Estrada and Vindas $[2]$.

In view of the simplicity and naturality of Theorem 1 , the known proof is somewhat indirect. The argument follows as a corollary of a more difficult result of Łojasiewicz, which is stated in Theorem 5. The first purpose of this paper is to give a short and direct proof. We then arrive at a formula of the indefinite integral of a distribution with a basepoint. In fact, we can reverse the usual direction of reasoning and use the arguments developed along these lines to give a different proof of Theorem 5 .

Theorem 5 is an example of a structure theorem, which is interesting in its own right and has a generalization involving the notion of the quasiasymptotic behavior [3]. In the last section, we study how variations of the definition of the value at a point lead to some other nice analogous structural results.

\section{A Proof of Theorem 1}

In order to fix our notation, we briefly recall the following elementary notions [4]. Suppose we have a continuous family of distributions $\left\{f_{u}\right\}_{u \in I}$ depending on a parameter $u$ in an interval $I$, meaning that $\left\langle f_{u}, \phi\right\rangle$ is continuous in $u$ for each $\phi \in \mathscr{D}$. If $\left\langle f_{u}, \phi\right\rangle$ is differentiable at $u_{0} \in I$ for each $\phi \in \mathscr{D}$, 
we say that $\left\{f_{u}\right\}_{u \in I}$ is differentiable with respect to $u$ at $u_{0}$ and define $\left.\partial_{u} f_{u}\right|_{u=u_{0}}$ by

$$
\left\langle\left.\partial_{u} f_{u}\right|_{u=u_{0}}, \phi\right\rangle=\left.\partial_{u}\left\langle f_{u}, \phi\right\rangle\right|_{u=u_{0}} .
$$

Evidently $\left.\partial_{u} f_{u}\right|_{u=u_{0}}$ is a distribution as it is the limit of distributions given by the difference quotients. Similarly, for $a, b \in I$, we define $\int_{a}^{b} f_{u} d u$ by

$$
\left\langle\int_{a}^{b} f_{u} d u, \phi\right\rangle=\int_{a}^{b}\left\langle f_{u}, \phi\right\rangle d u
$$

which is again a distribution, being the limit of distributions given by the Riemann sums. By pairing with test functions, it follows from the fundamental theorem of calculus that if $\left\{f_{u}\right\}_{u \in I}$ and $\left\{F_{u}\right\}_{u \in I}$ are continuous families of distributions with $\left.\partial_{u} F_{u}\right|_{u=u_{0}}=f_{u_{0}}$ for all $u_{0} \in I$, then, for any $a, b \in I$,

$$
\int_{a}^{b} f_{u} d u=F_{b}-F_{a}
$$

Let us note that, for any distribution $f(x) \in \mathscr{D}^{\prime}$, both $\{f(a x)\}_{a \in(-\infty, 0)}$ and $\{f(a x)\}_{a \in(0, \infty)}$ are continuous families of distributions. If $f$ is evaluable at $x_{0}=0$, namely, if $f(a x) \rightarrow$ $c$ as $a \rightarrow 0$, then $\{f(a x)\}_{a \in \mathbb{R}}$ becomes a continuous family of distributions if we define $f(0)=c$. Our argument uses this simple observation.

Proof of Theorem 1. Let $f=\partial F$ in $\mathscr{D}^{\prime}$ and suppose $f$ is evaluable at $x_{0}=0$. As seen above, $\{f(a x)\}_{a \in \mathbb{R}}$ is a continuous family of distributions and so is the family $\{x f(a x)\}_{a \in \mathbb{R}}$. It is trivial to verify that the family $\{F(a x)\}_{a \in(0, \infty)}$ is differentiable with respect to $a \in(0, \infty)$ with $\left.\partial_{a} F(a x)\right|_{a=a_{0}}=x f\left(a_{0} x\right)$. By (5), for $u, u_{0} \in(0, \infty)$,

$$
\int_{u}^{u_{0}} x f(a x) d a=F\left(u_{0} x\right)-F(u x) .
$$

The left-hand side is well defined for $u \in \mathbb{R}$ and gives a continuous family as $u$ ranges over the real line, and thus, taking the limit $u \rightarrow 0^{+}$on both sides, we see that $F(u x) \rightarrow L(x)$ as $u \rightarrow 0^{+}$for some $L(x) \in \mathscr{D}^{\prime}$. Applying $\partial_{x}$ gives $u f(u x) \rightarrow$ $L^{\prime}(x)$, but clearly $u f(u x) \rightarrow 0$. We conclude that $L(x)$ is a constant.

It also follows that if $F$ is a primitive of a distribution $f$ such that $f\left(x_{0}\right)=c$, the family $\left\{F\left(a x+x_{0}\right)\right\}_{a \in \mathbb{R}}$ is differentiable with respect to $a$ and we have $\left.\partial_{a} F\left(a x+x_{0}\right)\right|_{a=a_{0}}=$ $x f\left(a_{0} x+x_{0}\right)$. In particular, $\left.\partial_{a} F\left(a x+x_{0}\right)\right|_{a=0}=c x$.

\section{Distributions Integrable from a Basepoint}

In the preceding proof, it is clear that the assumption that $f$ is evaluable at $x_{0}$ was not entirely necessary. Let us say that $f \in \mathscr{D}^{\prime}$ is integrable from $x_{0}$ if the following two conditions hold.

(i) For $u_{0}>0, \int_{u}^{u_{0}} x f\left(a x+x_{0}\right) d a$ converges in $\mathscr{D}^{\prime}$ as $u \rightarrow$ $0^{+}$.

(ii) $a f\left(a x+x_{0}\right) \rightarrow 0$ in $\mathscr{D}^{\prime}$ as $a \rightarrow 0^{+}$.
By the same argument, this definition gives a necessary and sufficient condition for a primitive $F$ of $f$ to be evaluable at $x_{0}$. Indeed, if we set $x_{0}=0$, then (i) is equivalent to the existence of $L(x):=\lim _{u \rightarrow 0^{+}} F(u x)$. In this case, since $u f(u x) \rightarrow L^{\prime}(x)$ as $u \rightarrow 0^{+}$, (ii) is equivalent to $L(x)$ being a constant. We summarize this as follows.

Proposition 2. Let $F$ be a distribution and let $f=\partial F$. Then $F$ is evaluable at $x_{0}$ if and only if $f$ is integrable from $x_{0}$.

We denote by $\mathscr{D}_{x_{0}}^{\prime}$ the space of all distributions integrable from $x_{0}$. For $f \in \mathscr{D}_{x_{0}}^{\prime}$, we define a distribution $\int_{x_{0}}^{x+x_{0}} f$ by the formula

$$
\int_{x_{0}}^{x+x_{0}} f:=\lim _{u \rightarrow 0^{+}} \int_{u}^{1} x f\left(a x+x_{0}\right) d a .
$$

Let $F$ be a primitive of $f$. For any $u>0$,

$$
\int_{u}^{1} x f\left(a x+x_{0}\right) d a=F\left(x+x_{0}\right)-F\left(u x+x_{0}\right)
$$

and if $f$ is integrable from $x_{0}$, taking the limit $u \rightarrow 0^{+}$, we have

$$
\int_{x_{0}}^{x+x_{0}} f=F\left(x+x_{0}\right)-F\left(x_{0}\right)
$$

as $F\left(x_{0}\right)$ exists by Proposition 2. Replacing $x$ with $x-x_{0}$, we define the indefinite integral of $f \in \mathscr{D}_{x_{0}}^{\prime}$ with basepoint $x_{0}$ by

$$
\int_{x_{0}}^{x} f:=\lim _{u \rightarrow 0^{+}} \int_{u}^{1}\left(x-x_{0}\right) f\left(a x-a x_{0}+x_{0}\right) d a .
$$

It follows that we have $\int_{x_{0}}^{x} f=F(x)-F\left(x_{0}\right)$ and $\partial_{x} \int_{x_{0}}^{x} f=$ $f(x)$. We also note that $\int_{x_{0}}^{x} f$ is evaluable with value 0 at $x_{0}$.

It is easy to see that if $f_{n}$ is a sequence in $\mathscr{D}_{x_{0}}^{\prime}$, then $f_{n} \rightarrow$ $f$ in $\mathscr{D}^{\prime}$ for some $f \in \mathscr{D}_{x_{0}}^{\prime}$ does not imply $\int_{x_{0}}^{x} f_{n} \rightarrow \int_{x_{0}}^{x} f$ in general. In order to remedy this, we introduce the following notions.

Suppose $f_{n}$ is a sequence in $\mathscr{D}_{x_{0}}^{\prime}$. We say $f_{n}$ is bounded at $x_{0}$ if, for each $\phi \in \mathscr{D},\left\langle f_{n}\left(a x+x_{0}\right), \phi(x)\right\rangle$ is bounded independently of $n$ as well as of $a \in(0,1]$. Let us say $f_{n}$ converges boundedly to $f \in \mathscr{D}_{x_{0}}^{\prime}$ if $f_{n} \rightarrow f$ in $\mathscr{D}^{\prime}$ and $f_{n}-$ $f$ is eventually bounded at $x_{0}$. Finally, we say $f_{n}$ converges uniformly to $f \in \mathscr{D}_{x_{0}}^{\prime}$ if, for each $\phi \in \mathscr{D},\left\langle f_{n}\left(a x+x_{0}\right), \phi(x)\right\rangle$ converges to $\left\langle f\left(a x+x_{0}\right), \phi(x)\right\rangle$ uniformly in $a \in(0,1]$. Clearly, uniform convergence implies bounded convergence.

Lemma 3. If a sequence $f_{n}$ in $\mathscr{D}_{x_{0}}^{\prime}$ converges boundedly (resp., uniformly) to $f \in \mathscr{D}_{x_{0}}^{\prime}$, then the sequence $\int_{x_{0}}^{x} f_{n}$ converges boundedly (resp., uniformly) to $\int_{x_{0}}^{x} f$. 
Proof. Suppose $f_{n}$ in $\mathscr{D}_{x_{0}}^{\prime}$ converges to 0 with $f_{n}$ bounded at $x_{0}$, and let $F_{n}(x)=\int_{x_{0}}^{x} f_{n}$. We have

$$
\begin{aligned}
\left\langle F_{n}\left(a x+x_{0}\right), \phi(x)\right\rangle & =\lim _{u \rightarrow 0^{+}} \int_{u}^{1}\left\langle a x f_{n}\left(a b x+x_{0}\right), \phi(x)\right\rangle d b \\
& =\lim _{u \rightarrow 0^{+}} \int_{a u}^{a}\left\langle f_{n}\left(b x+x_{0}\right), x \phi(x)\right\rangle d b,
\end{aligned}
$$

which shows $\left\langle F_{n}\left(a x+x_{0}\right), \phi(x)\right\rangle$ is bounded independently of $n$ and of $a \in(0,1]$, and by taking the limit in $n$ under the integral sign, we see that $F_{n}$ converges boundedly to 0 . If $f_{n}$ in fact converges uniformly to 0 , the uniform convergence of $F_{n}$ is also apparent from the same expression.

Let us write $F_{n} \rightrightarrows F$ on $\Omega$ to mean that $F_{n}$ and $F$ are continuous functions on $\Omega$ such that $F_{n}$ converges to $F$ uniformly on $\Omega$. Let us also denote by $\int_{x_{0}}^{x}: \mathscr{D}_{x_{0}}^{\prime} \rightarrow \mathscr{D}_{x_{0}}^{\prime}$ the map that sends $f(x)$ to $\int_{x_{0}}^{x} f$.

Lemma 4. Let $f_{n}$, $f$ be distributions in $\mathscr{D}_{x_{0}}^{\prime}$. If $f_{n}$ converges boundedly to $f$, then, for every bounded open neighborhood $\Omega$ of $x_{0}$, there exists an integer $k \geq 0$ such that $\left(\int_{x_{0}}^{x}\right)^{k} f_{n} \rightrightarrows$ $\left(\int_{x_{0}}^{x}\right)^{k} f$ on $\Omega$.

Proof. Let $I$ be a compact interval containing $\Omega$. We can find $k \geq 0$ and a sequence of continuous functions $F_{n}, F$ on $I$ such that $f_{n}=\partial^{k} F_{n}, f=\partial^{k} F$ with $F_{n} \rightrightarrows F$ on $I$ (see [5]). Thus, $\left(\int_{x_{0}}^{x}\right)^{k} f_{n}$ and $F_{n}$ (resp., $\left(\int_{x_{0}}^{x}\right)^{k} f$ and $F$ ) differ by polynomials $P_{n}$ (resp., $P$ ) of degree $<k$ on I. By Lemma 3, $f_{n} \rightarrow f$ boundedly implies $\left(\int_{x_{0}}^{x}\right)^{k} f_{n} \rightarrow\left(\int_{x_{0}}^{x}\right)^{k} f$ in $\mathscr{D}^{\prime}$, and since $F_{n} \rightarrow F$ in $\mathscr{D}^{\prime}(\Omega)$, we have $P_{n} \rightarrow P$ in $\mathscr{D}^{\prime}(\Omega)$, which is the case only when $P_{n} \rightrightarrows P$ on $\Omega$. Hence $\left(\int_{x_{0}}^{x}\right)^{k} f_{n} \rightrightarrows\left(\int_{x_{0}}^{x}\right)^{k} f$ on $\Omega$.

\section{Structure Theorem of Lojasiewicz}

These ideas lead to a proof of another result of Łojasiewicz that we have already mentioned (cf. $[1,5,6])$. The proof given below seems illustrative in the sense that the implication in one direction is obtained by applying $\partial_{x}$ several times, and the converse is obtained by applying $\int_{x_{0}}^{x}$ several times.

Theorem 5 (Eojasiewicz). Let $f \in \mathscr{D}^{\prime}$. Then $f\left(a x+x_{0}\right) \rightarrow c$ as $a \rightarrow 0$ if and only if $f=\partial^{k} F$ for some $k \geq 0$, where $F$ is a continuous function near $x_{0}$ such that $\lim _{x \rightarrow x_{0}}(F(x) /(x-$ $\left.\left.x_{0}\right)^{k}\right)=c / k !$

Proof. Let $x_{0}=0$. If $f=\partial^{k} F$ and $F$ is continuous near 0 with $\lim _{x \rightarrow 0} F(x) / x^{k}=c / k$ !, then $F(a x) / a^{k} \rightarrow c x^{k} / k$ ! in $\mathscr{D}^{\prime}$ as $a \rightarrow 0$, and applying $\partial_{x}^{k}$ we obtain $f(a x) \rightarrow c$ in $\mathscr{D}^{\prime}$. Conversely, suppose $f(a x) \rightarrow c$ in $\mathscr{D}^{\prime}$ as $a \rightarrow 0$. Letting $f_{a}(x)=f(a x)$, it is easily observed that $f_{a}(x)$ converges boundedly (in fact, uniformly) to $c$ as $a \rightarrow 0$. By Lemma 4 , there exist a neighborhood $\Omega$ of 0 and $k \geq 0$ such that $\left(\int_{0}^{x}\right)^{k} f_{a} \rightrightarrows\left(\int_{0}^{x}\right)^{k} c=c x^{k} / k$ ! on $\Omega$. As $\left(\int_{0}^{x}\right)^{k} f_{a}=F(a x) / a^{k}$ if $F:=\left(\int_{0}^{x}\right)^{k} f$, we have $F(a x) / a^{k} \rightrightarrows c x^{k} / k$ ! as $a \rightarrow 0$. For any fixed $x \neq 0$ in $\Omega$, we have $F(a x) /(a x)^{k} \rightarrow c / k$ ! as $a \rightarrow 0$; namely, $\lim _{a \rightarrow 0} F(a) / a^{k}=c / k !$.

\section{Further Structure Theorems}

There are various notions of the value of a distribution at a point, some defined under stricter conditions with stronger properties while others applicable for more general distributions [7-11]. When a situation or an application demands some specific features from the evaluable distributions, one would like to know how the values that we obtain are associated with some structural qualities of the distributions. We now discuss some results of this type similar to Theorem 5 .

The works of Shiraishi and Itano give a notion of evaluation at a point with stricter properties than that of Łojasiewicz [7-9]. Let us call a sequence $\left(f_{n}\right)$ in $\mathscr{D}\left(\mathbb{R}^{d}\right)$ a $\delta$-sequence if there is a sequence of positive real numbers $\left(a_{n}\right) \rightarrow 0$ such that, $\forall n \in \mathbb{N}$,

(i) $f_{n}(x)=0$ for $|x| \geq a_{n}$,

(ii) $\int f_{n}=1$,

(iii) $\int\left|f_{n}\right|$ is bounded independently of $n \in \mathbb{N}$.

We say that a distribution $T \in \mathscr{D}^{\prime}\left(\mathbb{R}^{d}\right)$ has $\delta$-value $c \in \mathbb{C}$ at $x_{0} \in \mathbb{R}^{d}$ if

$$
\left\langle T, \tau_{x_{0}} f_{n}\right\rangle \longrightarrow c
$$

as $n \rightarrow \infty$ for all $\delta$-sequences $\left(f_{n}\right)$, where $\left(\tau_{x_{0}} f_{n}\right)(x)=f_{n}(x-$ $\left.x_{0}\right)$. In fact, we can restrict this condition to real nonnegative $\delta$-sequences (which are called $\delta$-sequences in, e.g., $[5,7]$ ) without affecting the definition. By the result in [7] (see also [12] for a proof based on ideas from nonstandard analysis), $T \in \mathscr{D}^{\prime}\left(\mathbb{R}^{d}\right)$ has $\delta$-value $c$ at $x_{0}$ if and only if it can be represented as an $L^{\infty}$-function near $x_{0}$ which is continuous at $x_{0}$ with value $c$. Thus, we have $T=c+\Psi$, with

$$
\underset{\left|x-x_{0}\right|<a}{\operatorname{ess} \sup _{\mid<}|\Psi(x)| \longrightarrow 0}
$$

as $a \rightarrow 0^{+}$. As this condition is quite strong, we can regard this as the most conservative notion of the value of a distribution at a point.

We can compare this with the previously discussed Łojasiewicz definition, as it is immediate that the Łojasiewicz value has the following sequential representation. A $\delta$ sequence $\left(f_{n}\right)$ of the form

$$
f_{n}(x)=a_{n}^{-d} f\left(\frac{x}{a_{n}}\right),
$$

where $f \in \mathscr{D}\left(\mathbb{R}^{d}\right)$ with $\int f=1$ and $a_{n}>0$ with $\left(a_{n}\right) \rightarrow 0$, is called a model sequence. One sees that a distribution $T$ has Łojasiewicz's value $c$ at $x_{0}$ if and only if $\left\langle T, \tau_{x_{0}} f_{n}\right\rangle \rightarrow c$ for all model sequences. A structural result given by Theorem 5 tells us that the condition imposed on $T$ is much weaker. 
In this section we find a continuous family of classes of distributions $\mathscr{D}_{p, x_{0}}$ for $1 \leq p<\infty$ such that, for any $1 \leq q \leq$ $p<\infty$,

$$
\left\{\begin{array}{c}
\text { Distributions } \\
\text { with } \delta \text {-value } \\
\text { at } x_{0}
\end{array}\right\} \subseteq \mathscr{D}_{q, x_{0}} \subseteq \mathscr{D}_{p, x_{0}} \subseteq\left\{\begin{array}{c}
\text { Distributions with } \\
\text { Łojasiewicz's value } \\
\text { at } x_{0}
\end{array}\right\}
$$

with analogous structural results involving $L^{p}$ functions. These classes of distributions can be defined sequentially in a natural way.

Definition 6. Let $1 \leq p<\infty$ be fixed. A sequence $\left(f_{n}\right)$ in $\mathscr{D}\left(\mathbb{R}^{d}\right)$ is called a $\delta_{p}$-sequence if there exists a sequence of positive real numbers $\left(a_{n}\right) \rightarrow 0$ such that, $\forall n \in \mathbb{N}$,

(i) $f_{n}(x)=0$ for $|x| \geq a_{n}$,

(ii) $\int f_{n}=1$,

(iii) $a_{n}^{d(p-1)} \int\left|f_{n}\right|^{p}$ is bounded independently of $n \in \mathbb{N}$.

A distribution $T \in \mathscr{D}^{\prime}\left(\mathbb{R}^{d}\right)$ is said to have $\delta_{p}$-value $c \in \mathbb{C}$ at $x_{0} \in \mathbb{R}^{d}$ if

$$
\left\langle T, \tau_{x_{0}} f_{n}\right\rangle \longrightarrow c
$$

as $n \rightarrow \infty$ for all $\delta_{p}$-sequences $\left(f_{n}\right)$.

Remark 7. In the above definition, we will say that $\left(a_{n}\right)$ is a contracting sequence of $\left(f_{n}\right)$.

For any $1 \leq q \leq p \leq \infty$, we have (e.g., [13]) that if $\Omega \subseteq$ $\mathbb{R}^{d}$ is a nonempty open subset of finite measure $\mu(\Omega)$ and if $f \in L^{p}(\Omega)$, then $f \in L^{q}(\Omega)$ and

$$
\|f\|_{L^{q}(\Omega)} \leq \mu(\Omega)^{1 / q-1 / p}\|f\|_{L^{p}(\Omega)} .
$$

Let $1 \leq q \leq p<\infty$, and suppose $\left(f_{n}\right)$ is a $\delta_{p}$-sequence, with a contracting sequence $\left(a_{n}\right) \rightarrow 0$. From (17) we obtain

$$
a_{n}^{-d / q}\left(\int\left|f_{n}\right|^{q}\right)^{1 / q} \leq a_{n}^{-d / p}\left(\int\left|f_{n}\right|^{p}\right)^{1 / p}
$$

and multiplying both sides by $a_{n}^{d}$ gives

$$
\left(a_{n}^{d(q-1)} \int\left|f_{n}\right|^{q}\right)^{1 / q} \leq\left(a_{n}^{d(p-1)} \int\left|f_{n}\right|^{p}\right)^{1 / p}
$$

which shows that $\left(f_{n}\right)$ is also a $\delta_{q}$-sequence. Therefore, if a distribution $T$ has $\delta_{q}$-value $c$ at $x_{0}$, then it has the same $\delta_{p^{-}}$ value at $x_{0}$. This will also follow from Theorem 10 (iii), as we have, since $1<p^{\prime} \leq q^{\prime} \leq \infty$,

$$
a^{-d / p^{\prime}}\|\Psi\|_{L^{p^{\prime}}\left(B_{x_{0}}(a)\right)} \leq a^{-d / q^{\prime}}\|\Psi\|_{L^{q^{\prime}}\left(B_{x_{0}}(a)\right)}
$$

by (17). Hence, the condition of a distribution having $\delta_{p}$-value at a point becomes less restrictive as $p$ increases. As any model sequence is a $\delta_{p}$-sequence for all $1 \leq p<\infty$, if $T \in \mathscr{D}\left(\mathbb{R}^{d}\right)$ has $\delta_{p}$-value $c$ at $x_{0} \in \mathbb{R}^{d}$ for some $p$, then it has the same value $c$ at $x_{0}$ in the sense of Łojasiewicz.
For a nonempty open set $\Omega \subseteq \mathbb{R}^{d}$, we let $\mathscr{D}_{\mathbb{R}}(\Omega) \subseteq$ $\mathscr{D}(\Omega)$ be the subspace of all real valued test functions and let $\mathscr{D}(\Omega)_{+} \subseteq \mathscr{D}_{\mathbb{R}}(\Omega)$ (resp., $\mathscr{D}(\Omega)_{-} \subseteq \mathscr{D}_{\mathbb{R}}(\Omega)$ ) be the subset of all nonnegative (resp., nonpositive) test functions. Let

$$
\mathscr{D}_{p}^{1}(\Omega) \subseteq \mathscr{D}(\Omega)
$$

be the subset consisting of $f$ such that $\int|f|^{p}=1$, and let

$$
\mathscr{D}_{p}^{1}(\Omega)_{+}=\mathscr{D}_{p}^{1}(\Omega) \cap \mathscr{D}(\Omega)_{+} .
$$

For $\Psi \in \mathscr{D}^{\prime}(\Omega)$, we define

$$
\begin{aligned}
\|\Psi\|_{\mathscr{D}_{p}^{\prime}(\Omega)} & =\sup _{f \in \mathscr{D}_{p}^{1}(\Omega)}|\langle\Psi, f\rangle|, \\
\|\Psi\|_{\mathscr{D}_{p}^{\prime}(\Omega)_{+}} & =\sup _{f \in \mathscr{D}_{p}^{1}(\Omega)_{+}}|\langle\Psi, f\rangle|,
\end{aligned}
$$

taking values in $[0, \infty]$. We then have the following simple estimate.

Lemma 8. We have

$$
\|\Psi\|_{\mathscr{D}_{p}^{\prime}(\Omega)_{+}} \leq\|\Psi\|_{\mathscr{D}_{p}^{\prime}(\Omega)} \leq 4\|\Psi\|_{\mathscr{D}_{p}^{\prime}(\Omega)_{+}} .
$$

Proof. The first inequality follows trivially since $\mathscr{D}_{p}^{1}(\Omega)_{+} \subseteq$ $\mathscr{D}_{p}^{1}(\Omega)$. In order to see the second inequality, suppose $f \in$ $\mathscr{D}_{\mathbb{R}}(\Omega)$. We can write $f=f_{+}+f_{-}$, where $f_{+}(x)=\max \{f(x)$, $0\}$ and $f_{-}(x)=\min \{f(x), 0\}$ for $x \in \Omega$. As $f_{+}$and $f_{-}$are compactly supported continuous functions, we can find $f_{1} \epsilon$ $\mathscr{D}(\Omega)_{+}$(resp., $\left.f_{2} \in \mathscr{D}(\Omega)_{-}\right)$that is as close as we want to $f_{+}$ (resp., $f_{-}$) in the $L^{p}$-norm, such that $f=f_{1}+f_{2}$. Hence, from

$$
\begin{aligned}
|\langle\Psi, f\rangle| & \leq\left|\left\langle\Psi, f_{1}\right\rangle\right|+\left|\left\langle\Psi, f_{2}\right\rangle\right| \\
& \leq\|\Psi\|_{\mathscr{D}_{p}^{\prime}(\Omega)_{+}}\left(\left\|f_{1}\right\|_{L^{p}(\Omega)}+\left\|f_{2}\right\|_{L^{p}(\Omega)}\right),
\end{aligned}
$$

since $\left\|f_{ \pm}\right\|_{L^{p}(\Omega)} \leq\|f\|_{L^{p}(\Omega)}$, we have

$$
|\langle\Psi, f\rangle| \leq 2\|\Psi\|_{\mathscr{D}_{p}^{\prime}(\Omega)_{+}}\|f\|_{L^{p}(\Omega)} .
$$

By (26), if $f \in \mathscr{D}(\Omega)$, then since we have $\|\operatorname{Re}(f)\|_{L^{p}(\Omega)} \leq$ $\|f\|_{L^{p}(\Omega)}$ and $\|\operatorname{Im}(f)\|_{L^{p}(\Omega)} \leq\|f\|_{L^{p}(\Omega)}$,

$$
\begin{aligned}
|\langle\Psi, f\rangle| & \leq|\langle\Psi, \operatorname{Re}(f)\rangle|+|\langle\Psi, \operatorname{Im}(f)\rangle| \\
& \leq 4\|\Psi\|_{\mathscr{D}_{p}^{\prime}(\Omega)_{+}}\|f\|_{L^{p}(\Omega)} .
\end{aligned}
$$

Suppose $\|\Psi\|_{\mathscr{D}_{p}^{\prime}(\Omega)}<\infty$ for some $1 \leq p<\infty$. Since $\mathscr{D}(\Omega)$ is dense in $L^{p}(\Omega), \Psi$ extends to a continuous functional on $L^{p}(\Omega)$ and lies in the strong dual of $L^{p}(\Omega)$, which is isometric to $L^{p^{\prime}}(\Omega)[13]$. We thus have $\Psi \in L^{p^{\prime}}(\Omega)$ and $\|\Psi\|_{\mathscr{D}_{p}^{\prime}(\Omega)}=$ $\|\Psi\|_{L^{p^{\prime}}(\Omega)}$.

Lemma 9. Let $\left(a_{n}\right)$ be a sequence of positive real numbers such that $\left(a_{n}\right) \rightarrow 0$. Suppose we have two sequences $\left(f_{n}\right)$ and $\left(g_{n}\right)$ in $\mathscr{D}\left(\mathbb{R}^{d}\right)$ such that both $a_{n}^{d(p-1)} \int\left|f_{n}\right|^{p}$ and $a_{n}^{d(p-1)} \int\left|g_{n}\right|^{p}$ are bounded independently of $n \in \mathbb{N}$. Then $a_{n}^{d(p-1)} \int\left|f_{n}+g_{n}\right|^{p}$ is bounded independently of $n \in \mathbb{N}$. 
Proof. By multiplying by $a_{n}^{d(p-1)}$ on both sides of Minkowski's inequality for $f_{n}$ and $g_{n}$, we obtain

$$
\begin{aligned}
& a_{n}^{d(p-1)} \int\left|f_{n}+g_{n}\right|^{p} \\
& \quad \leq\left(\left(a_{n}^{d(p-1)} \int\left|f_{n}\right|^{p}\right)^{1 / p}+\left(a_{n}^{d(p-1)} \int\left|g_{n}\right|^{p}\right)^{1 / p}\right)^{p},
\end{aligned}
$$

from which the lemma follows.

We can now give a structure theorem on our notion of $\delta_{p}-$ value of a distribution. The only tricky part of the following argument seems to be that our definition is unaffected even if we only restrict ourselves to real nonnegative $\delta_{p}$-sequences (Theorem 10 (ii))

Theorem 10. Let $T \in \mathscr{D}^{\prime}\left(\mathbb{R}^{d}\right)$. Then, the following statements are equivalent.

(i) $T$ has $\delta_{p}$-value $c \in \mathbb{C}$ at $x_{0} \in \mathbb{R}^{d}$.

(ii) $\left\langle T, \tau_{x_{0}} f_{n}\right\rangle \rightarrow c$ as $n \rightarrow \infty$ for all $\delta_{p}$-sequences $\left(f_{n}\right)$ such that $f_{n} \geq 0$.

(iii) $T=c+\Psi$, where $\Psi$ can be represented as an $L^{p^{\prime}}$ function in some open ball $B_{x_{0}}(a)$ of radius $a>0$ around $x_{0}$, and

$$
a^{-d / p^{\prime}}\|\Psi\|_{L^{p^{\prime}\left(B_{x_{0}}(a)\right)}} \longrightarrow 0
$$

as $a \rightarrow 0^{+}$, where $p^{\prime}=p /(p-1) \in(1, \infty]$ is the Hölder conjugate of $p$.

Proof. As the implication (i) $\Rightarrow$ (ii) is immediate, it only remains to show (ii) $\Rightarrow$ (iii) $\Rightarrow$ (i).

Let us assume (ii). It suffices to consider the special case $x_{0}=0$. Let $T$ be a distribution such that $\left(\left\langle T, f_{n}\right\rangle\right) \rightarrow c$ for all nonnegative $\delta_{p}$-sequences $\left(f_{n}\right)$. For $\Psi=T-c$, since $\int f_{n}=1$ and $\left\langle T-c, f_{n}\right\rangle=\left\langle T, f_{n}\right\rangle-c$, we have $\left(\left\langle T, f_{n}\right\rangle\right) \rightarrow c$ if and only if $\left(\left\langle\Psi, f_{n}\right\rangle\right) \rightarrow 0$. We now claim that

$$
a^{-d / p^{\prime}}\|\Psi\|_{\mathscr{D}_{p}^{\prime}\left(B_{0}(a)\right)_{+}} \longrightarrow 0
$$

as $a \rightarrow 0^{+}$. Otherwise, for some $\varepsilon_{0}>0$, we can find a sequence of positive real numbers $\left(a_{n}\right) \rightarrow 0$ and functions $g_{n} \in \mathscr{D}_{p}^{1}\left(B_{0}\left(a_{n}\right)\right)_{+}$such that $a_{n}^{-d / p^{\prime}}\left|\left\langle\Psi, g_{n}\right\rangle\right| \geq \varepsilon_{0}$ for all $n \in \mathbb{N}$. We note

$$
a_{n}^{d(p-1)} \int\left(a_{n}^{-d / p^{\prime}} g_{n}\right)^{p}=a_{n}^{d(p-1)} a_{n}^{-d(p-1)} \int g_{n}^{p}=1,
$$

and, in particular, it is bounded independently of $n \in \mathbb{N}$. Applying inequality (19) to the functions $a_{n}^{-d / p^{\prime}} g_{n}$ (with $q=$ 1), we obtain

$$
\int a_{n}^{-d / p^{\prime}} g_{n} \leq 1
$$

for all $n \in \mathbb{N}$. Let $\left(h_{n}\right)$ be any fixed nonnegative $\delta_{p}$-sequence of which $\left(a_{n}\right)$ is a contracting sequence, such as a nonnegative model sequence. We let

$$
f_{n}=a_{n}^{-d / p^{\prime}} g_{n}+b_{n} h_{n}
$$

where $b_{n}=1-\int a_{n}^{-d / p^{\prime}} g_{n} \in[0,1)$. Observe that $f_{n} \geq 0$ with $\int f_{n}=1$, and applying Lemma 9 to the sequences $a_{n}^{-d / p^{\prime}} g_{n}$ and $b_{n} h_{n}$, we see that $\left(f_{n}\right)$ is in fact a nonnegative $\delta_{p}$-sequence. Thus, we must have

$$
\left(\left\langle\Psi, f_{n}\right\rangle\right) \longrightarrow 0
$$

But as $\left\langle\Psi, f_{n}\right\rangle=a_{n}^{-d / p^{\prime}}\left\langle\Psi, g_{n}\right\rangle+b_{n}\left\langle\Psi, h_{n}\right\rangle$, the fact that $\left(b_{n}\langle\Psi\right.$, $\left.\left.h_{n}\right\rangle\right) \rightarrow 0$ implies $\left(a_{n}^{-d / p^{\prime}}\left\langle\Psi, g_{n}\right\rangle\right) \rightarrow 0$, a contradiction. Hence, (30) follows, which implies (iii) by Lemma 8 and the paragraph following it.

Lastly, we assume that (iii) holds for $x_{0}=0$. Let $\left(f_{n}\right)$ be a $\delta_{p}$-sequence with a contracting sequence $\left(a_{n}\right) \rightarrow 0$. By Hölder's inequality,

$$
\begin{aligned}
\left|\left\langle\Psi, f_{n}\right\rangle\right| & \leq\|\Psi\|_{L^{p^{\prime}}\left(B\left(a_{n}\right)\right)}\left\|f_{n}\right\|_{L^{p}\left(B\left(a_{n}\right)\right)} \\
& =a_{n}^{-d / p^{\prime}}\|\Psi\|_{L^{p^{\prime}}\left(B\left(a_{n}\right)\right)}\left(a_{n}^{d(p-1)} \int\left|f_{n}\right|^{p}\right)^{1 / p} \longrightarrow 0
\end{aligned}
$$

as $n \rightarrow \infty$, and (i) follows.

It is often useful to relate a given notion of a value at a point, usually defined through the pairing of a distribution with test functions, to a statement revealing the internal structure of the distribution. One such result is Theorem 5, and the above theorem gives some others.

\section{Conflict of Interests}

The author declares that there is no conflict of interests regarding the publication of this paper.

\section{Acknowledgment}

The author wishes to thank the referees for insightful comments and valuable advice on the presentation.

\section{References}

[1] S. Łojasiewicz, "Sur la valeur et la limite d'une distribution en un point," Studia Mathematica, vol. 16, pp. 1-36, 1957.

[2] R. Estrada and J. Vindas, "A general integral," Dissertationes Mathematicae, vol. 483, pp. 1-49, 2012.

[3] J. Vindas and S. Pilipović, "Structural theorems for quasiasymptotics of distributions at the origin," Mathematische Nachrichten, vol. 282, no. 11, pp. 1584-1599, 2009.

[4] I. M. Gel'fand and G. E. Shilov, Generalized Functions. Vol. 1: Properties and Operations, Academic Press, New York, NY, USA, 1964.

[5] P. Antosik, J. Mikusiński, and R. Sikorski, Theory of Distributions. The Sequential Approach, Elsevier Scientific, Amsterdam, The Netherlands, 1973.

[6] S. Łojasiewicz, "Sur la fixation des variables dans une distribution," Studia Mathematica, vol. 17, pp. 1-64, 1958.

[7] R. Shiraishi and M. Itano, "On the multiplicative products of distributions," Journal of Science of the Hiroshima University AI: Mathematics, vol. 28, pp. 223-235, 1964. 
[8] M. Itano, "On the multiplicative products of distributions," Journal of Science of the Hiroshima University A-I: Mathematics, vol. 29, pp. 51-74, 1965.

[9] R. Shiraishi, "On the value of distributions at a point and the multiplicative products," Journal of Science of the Hiroshima University A-I: Mathematics, vol. 31, pp. 89-104, 1967.

[10] M. D. Cohen, "On the value of a distribution at a point," Mathematische Zeitschrift, vol. 122, pp. 101-103, 1971.

[11] J. Mikusiński, “On the value of a distribution at a point," Bulletin de l'Académie Polonaise des Sciences. Série des Sciences Mathématiques, Astronomiques et Physiques, vol. 8, pp. 681-683, 1960.

[12] H. Vernaeve and J. Vindas, "Characterization of distributions having a value at a point in the sense of Robinson," Journal of Mathematical Analysis and Applications, vol. 396, no. 1, pp. 371374, 2012.

[13] F. Trèves, Topological Vector Spaces, Distributions and Kernels, Academic Press, New York, NY, USA, 1967. 


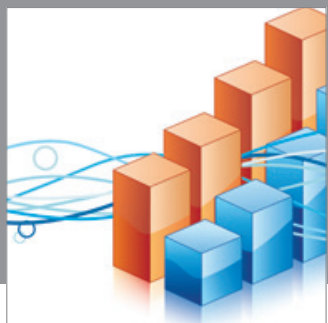

Advances in

Operations Research

mansans

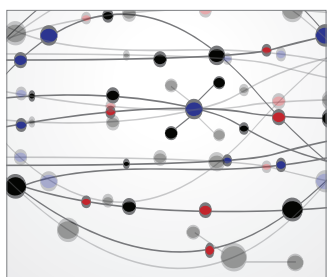

The Scientific World Journal
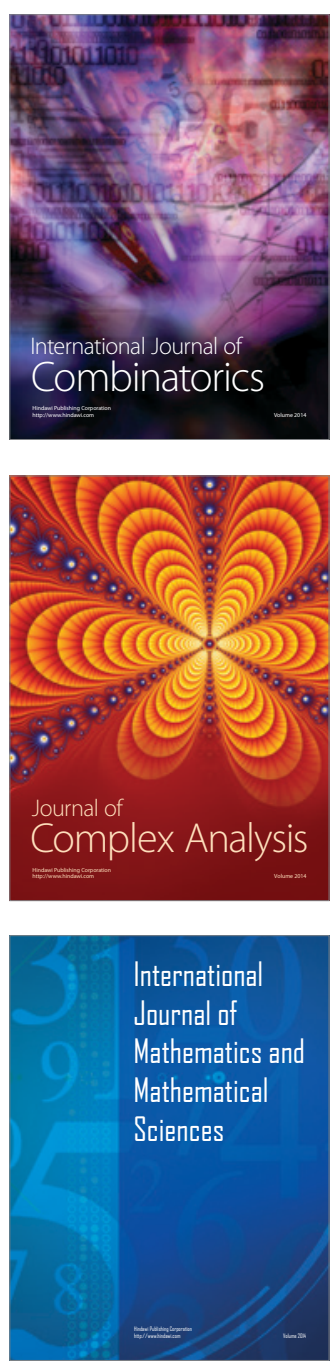
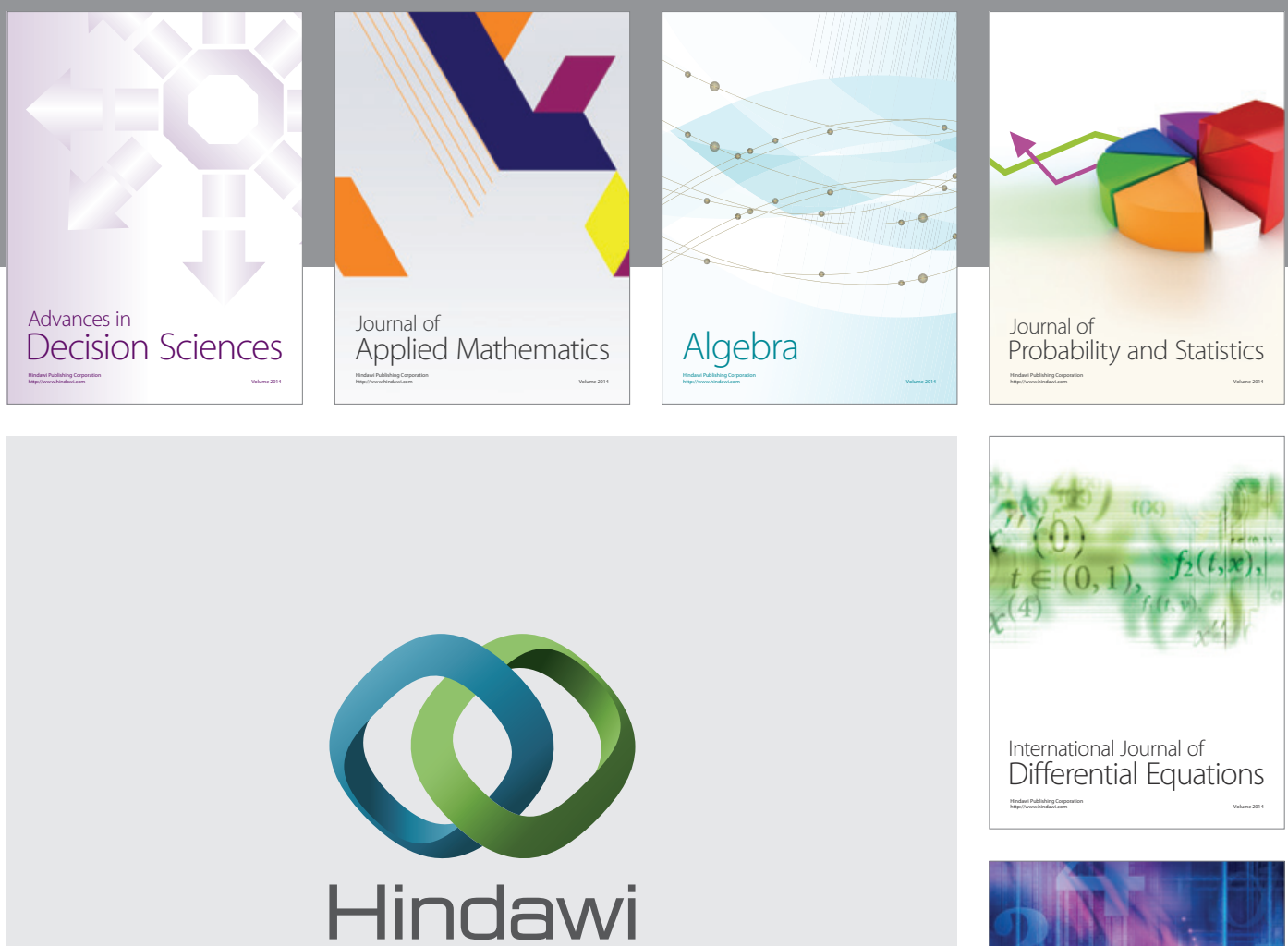

Submit your manuscripts at http://www.hindawi.com
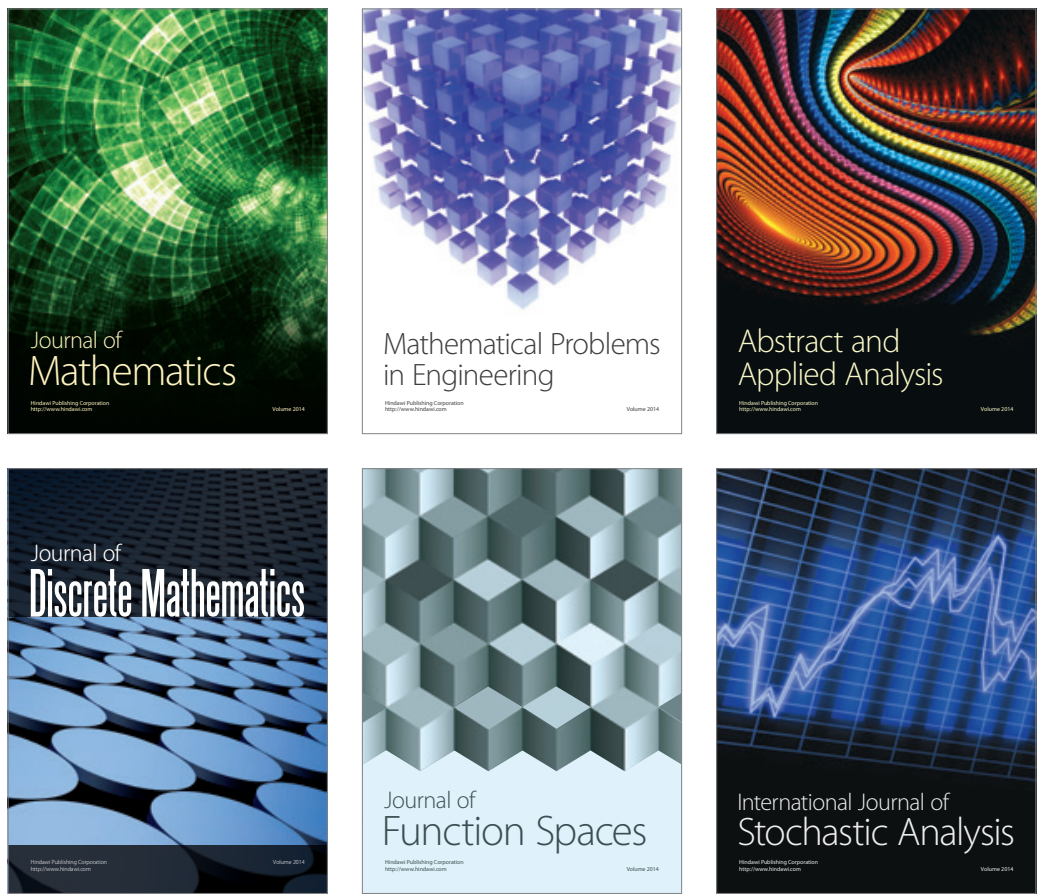

Journal of

Function Spaces

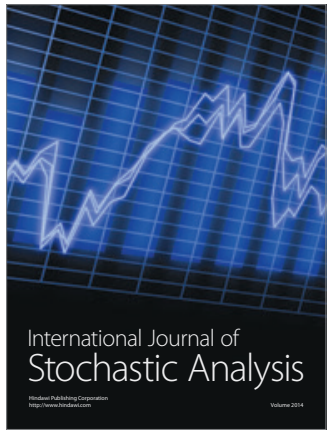

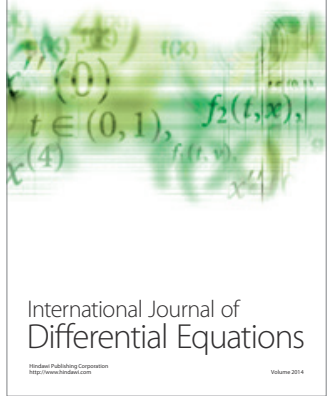
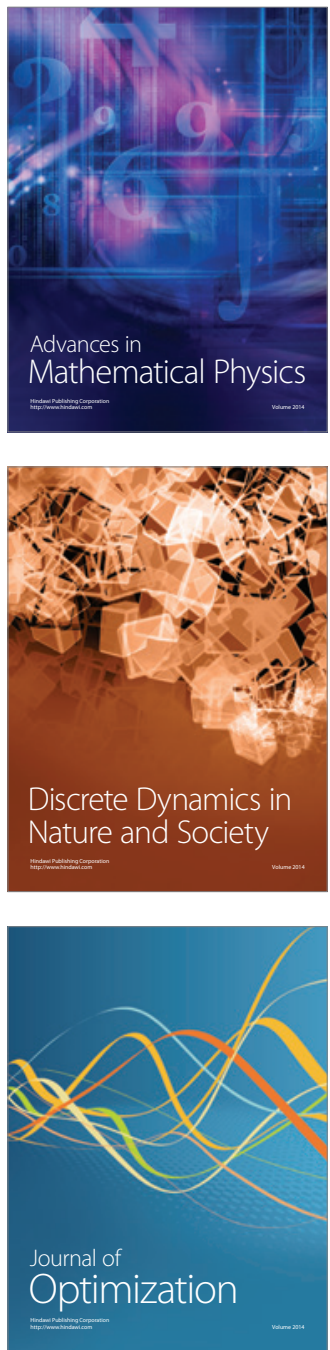